\title{
LINE GRAPHS OF COMPLEX UNIT GAIN GRAPHS WITH LEAST EIGENVALUE $-2^{*}$
}

\author{
FRANCESCO BELARDO $^{\dagger}$ AND MAURIZIO BRUNETTI ${ }^{\dagger}$
}

\begin{abstract}
Let $\mathbb{T}$ be the multiplicative group of complex units, and let $\mathcal{L}(\Phi)$ denote a line graph of a $\mathbb{T}$-gain graph $\Phi$. Similarly to what happens in the context of signed graphs, the real number $\min \operatorname{Spec}(A(\mathcal{L}(\Phi))$, that is, the smallest eigenvalue of the adjacency matrix of $\mathcal{L}(\Phi)$, is not less than -2 . The structural conditions on $\Phi$ ensuring that $\min \operatorname{Spec}(A(\mathcal{L}(\Phi))=-2$ are identified. When such conditions are fulfilled, bases of the -2-eigenspace are constructed with the aid of the star complement technique.
\end{abstract}

Key words. Complex unit gain graph, Line graph, Subdivision graph, Oriented gain graph, Voltage graph, Star complement technique.

AMS subject classifications. 05C22, 05C50, 05C 76, 05C25.

1. Introduction. Let $\Gamma$ be a simple graph with vertex set $V(\Gamma)=\left\{v_{1}, v_{2}, \ldots, v_{n}\right\}$, and let $\vec{E}(\Gamma)$ be the set of oriented edges. Each edge of $\Gamma$ determines two different elements in $\vec{E}(\Gamma)$. Namely, if $v_{i}$ and $v_{j}$ are adjacent in $\Gamma$, we find in $\vec{E}(\Gamma)$ the oriented edge $e_{i j}$ which goes from $v_{i}$ to $v_{j}$, and $e_{j i}$ going in the opposite direction. Given any group $\mathfrak{G}$, a $(\mathfrak{G}$ - $)$ gain graph is a triple $\Phi=(\Gamma, \mathfrak{G}, \gamma)$ consisting of an underlying graph $\Gamma$, the gain group $\mathfrak{G}$, and a map $\gamma: \vec{E}(\Gamma) \rightarrow \mathfrak{G}$ such that $\gamma\left(e_{i j}\right)=\gamma\left(e_{j i}\right)^{-1}$ called the gain function. The gain graph $\Phi$ is said to be balanced if for every direct cycle $\vec{C}=e_{i_{1} i_{2}} \cdots e_{i_{k} i_{1}}$ in $\Gamma$ (if any), we have $\gamma\left(e_{i_{1} i_{2}}\right) \gamma\left(e_{i_{2} i_{3}}\right) \cdots \gamma\left(e_{i_{k} i_{1}}\right)=1$. A gain graph is said to be unbalanced if it is not balanced. Most of the concepts defined for simple graphs directly extend to gain graphs. For instance, we say that a gain graph $\Phi=(\Gamma, \mathfrak{G}, \gamma)$ is of order $n$ and size $m$ if its underlying graph $\Gamma$ has $n$ vertices and $m$ edges; moreover, we say that a gain graph $(\Gamma, \mathfrak{G}, \gamma)$ is $k$-cyclic if the underlying graph $\Gamma$ is connected and $|E(\Gamma)|=|V(\Gamma)|+k-1$. As usual, the words unicyclic and bicyclic stand as synonyms for 1-cyclic and 2-cyclic, respectively.

Gain graphs (also known in the literature as voltage graphs) are studied in many research areas (see [21] and the annotated bibliography [22]).

In particular, a complex unit gain graph is a $\mathfrak{G}$-gain graph with $\mathfrak{G}$ being equal to the multiplicative group $\mathbb{T}$ of all complex numbers with norm 1 . The theory of complex unit gain graphs embodies those of signed graphs and mixed graphs (as defined in [14]). In fact, a signed graph (resp. mixed graph) can be seen as a particular $\mathbb{T}$-gain graph with gains in the subset $\{ \pm 1\}$ (resp. $\{1, \pm i\})$ of $\mathbb{T}$.

Over the last decade, there has been a growing interest for the study of matrices and eigenvalues associated with $\mathbb{T}$-gain graphs. For instance, in [17], Reff studied many properties of the adjacency and the Laplacian matrix of $\mathbb{T}$-gain graphs. Further spectral results concerning $\mathbb{T}$-gain graphs have been obtained in $[2,16]$ (where $\mathbb{T}$-gain graphs are called weighted directed graphs). More recently, in [4] the authors figured out how the least Laplacian eigenvalue of a $\mathbb{T}_{4}$-gain graph (i.e. a $\mathbb{T}$-gain graph with gains in $\{ \pm 1, \pm i\}$ ) is

${ }^{*}$ Received by the editors on March 11, 2020. Accepted for publication on October 17, 2020. Handling Editor: Froilán Dopico. Corresponding Author: Maurizio Brunetti.

${ }^{\dagger}$ Department of Mathematics and Applications, University of Naples 'Federico II', Italy (francesco.belardo2@unina.it, maurizio.brunetti@unina.it). 
related to its frustration index and number. Moreover, Godsil-McKay-like switchings have been described in [3] for the purpose of identifying pairs of non-isomorphic cospectral $\mathbb{T}$-gain graphs.

In [18], Reff introduced a notion of orientation for gain graphs in order to provide a suitable setting to build up line graphs of gain graphs. In the wake of his seminal ideas, the authors of this paper specialized in [1] Reff's results to $\mathbb{T}_{4}$-gain graphs.

The starting point of this paper is Theorem 2.14, which extends Theorem 4 in [1] to complex unit gain graphs. It turns out that, for every complex unit gain graph $\Phi$, the minimum possible eigenvalue for the adjacency matrix of an associated line graph $\mathcal{L}(\Phi)$ is -2 . We prove that such minimum is attained whenever $\Phi$ has a connected component which is neither a tree nor a balanced unicyclic gain graph. In these cases, we study the -2-eigenspace, detecting a basis by using the star complement technique and generalizing the routine successfully applied in the past to simple graphs (see $[10,11,12])$ and to signed graphs (see $[5,6]$ ).

The remainder of the paper is organized as follows. In Section 2, we recall some background theory on $\mathbb{T}$-gain graphs, the star complement technique, and the basic properties of line graphs associated with $\mathbb{T}$-gain graphs. In Section 3, we explicitly compute the components of -2 -eigenvectors in all cases when -2 belongs to the adjacency spectrum of the line graph $\mathcal{L}(\Phi)$. The final section contains two examples.

\section{Preliminaries.}

2.1. Gain graphs. From now on, a $\mathbb{T}$-gain graph will be simply denoted by $\Phi=(\Gamma, \gamma)$. Given a $\mathbb{T}$-gain graph $\Phi=(\Gamma, \gamma)$ of order $n$ and size $m$, we adopt the notation

$$
V(\Gamma)=\left\{v_{1}, \ldots, v_{n}\right\} \quad \text { and } \quad \mathrm{E}(\Gamma)=\left\{e_{1}, \ldots, e_{m}\right\},
$$

for the set of vertices and the set of (unoriented) edges of $\Gamma$, respectively.

Let $M_{m, n}(\mathbb{C})$ be the set of $m \times n$ complex matrices. For a matrix $A=\left(a_{i j}\right) \in M_{m, n}(\mathbb{C})$, we denote by $A^{*}=\left(a_{i j}^{*}\right) \in M_{n, m}(\mathbb{C})$ its conjugate (or Hermitian) transpose, that is, $a_{i j}^{*}=\bar{a}_{j i}$.

The adjacency matrix $A(\Phi)=\left(a_{i j}\right) \in M_{n, n}(\mathbb{C})$ of a $\mathbb{T}$-gain graph $\Phi=(\Gamma, \gamma)$ is defined by

$$
a_{i j}= \begin{cases}\gamma\left(e_{i j}\right) & \text { if } v_{i} \text { is adjacent to } v_{j}, \\ 0 & \text { otherwise. }\end{cases}
$$

If $v_{i}$ is adjacent to $v_{j}$, then $a_{i j}=\gamma\left(e_{i j}\right)=\gamma\left(e_{j i}\right)^{-1}=\overline{\gamma\left(e_{j i}\right)}=\bar{a}_{j i}$. Consequently, $A(\Phi)$ is Hermitian and its eigenvalues $\lambda_{1}(\Phi) \geqslant \cdots \geqslant \lambda_{n}(\Phi)$ are real. The Laplacian matrix $L(\Phi)$ is defined as $D(\Gamma)-A(\Phi)$, where $D(\Gamma)$ stands for the diagonal matrix of vertex degrees of $\Gamma$. Therefore, $L(\Phi)$ is also Hermitian. According to [17], the matrix $L(\Phi)$ is positive semidefinite, and all its eigenvalues $\mu_{1}(\Phi) \geqslant \cdots \geqslant \mu_{n}(\Phi)$ are nonnegative. We write $\phi(\Phi, x)$ and $\psi(\Phi, x)$ to denote the characteristic polynomial of $A(\Phi)$ and $L(\Phi)$, respectively. By definition, the spectrum $\operatorname{Spec}(A(\Phi))$ (resp. $\operatorname{Spec}(L(\Phi))$ ) is the multiset of eigenvalues of $A(\Phi)$ (resp. of $L(\Phi)$ ). For every eigenvalue $\lambda$ of $A(\Phi)$, the corresponding eigenspace is denoted by $\mathcal{E}_{\Phi}(\lambda)$.

A switching function of a given $\mathbb{T}$-gain graph $\Phi$ is any map $\zeta: V(\Gamma) \rightarrow \mathbb{T}$. Switching the $\mathbb{T}$-gain graph $\Phi=(\Gamma, \gamma)$ means replacing $\gamma$ by $\gamma^{\zeta}$, where $\gamma^{\zeta}\left(e_{i j}\right)=\zeta\left(v_{i}\right)^{-1} \gamma\left(e_{i j}\right) \zeta\left(v_{j}\right)$ and obtaining the new $\mathbb{T}$-gain graph $\Phi^{\zeta}=\left(\Gamma, \gamma^{\zeta}\right)$. We say that $\Phi_{1}=\left(\Gamma, \gamma_{1}\right)$ and $\Phi_{2}=\left(\Gamma, \gamma_{2}\right)$ (and their corresponding gain functions) are switching equivalent if there exists a switching function $\zeta$ such that $\Phi_{2}=\Phi_{1}^{\zeta}$. By writing $\Phi_{1} \sim \Phi_{2}$ or $\gamma_{1} \sim \gamma_{2}$, we mean that $\Phi_{1}$ and $\Phi_{2}$ are switching equivalent. 
Electronic Journal of Linear Algebra, ISSN 1081-3810

To each switching function $\zeta$, we associate a diagonal matrix $D(\zeta)=\operatorname{diag}\left(\zeta\left(v_{1}\right), \ldots, \zeta\left(v_{n}\right)\right)$. Note that

$$
A\left(\Phi_{2}\right)=D(\zeta)^{*} A\left(\Phi_{1}\right) D(\zeta) \quad \text { and } \quad L\left(\Phi_{2}\right)=D(\zeta)^{*} L\left(\Phi_{1}\right) D(\zeta)
$$

Therefore, given any pair $\left(\Phi_{1}, \Phi_{2}\right)$ of switching equivalent $\mathbb{T}$-gain graphs, we get the following equality between their spectra:

$$
\operatorname{Spec}\left(A\left(\Phi_{1}\right)\right)=\operatorname{Spec}\left(A\left(\Phi_{2}\right)\right) \quad \text { and } \quad \operatorname{Spec}\left(L\left(\Phi_{1}\right)\right)=\operatorname{Spec}\left(L\left(\Phi_{2}\right)\right)
$$

One of the key notions in the theory of gain graphs (and of the more general theory of biased graphs) is the property of balance (see [9, 21, 23]). An oriented edge $e_{i_{h} i_{k}} \in \vec{E}(\Gamma)$ is said to be neutral for $\Phi=(\Gamma, \gamma)$ if $\gamma\left(e_{i_{h} i_{k}}\right)=1$. Similarly, the walk $W=e_{i_{1} i_{2}} e_{i_{2} i_{3}} \cdots e_{i_{l-1} i_{l}}$ is said to be neutral if its gain

$$
\gamma(W):=\gamma\left(e_{i_{1} i_{2}}\right) \gamma\left(e_{i_{2} i_{3}}\right) \cdots \gamma\left(e_{i_{l-1} i_{l}}\right)
$$

is equal to 1 . We write $(\Gamma, 1)$ for the $\mathbb{T}$-gain graph with all neutral edges.

An edge set $S \subseteq E$ is said to be balanced if every directed cycle $\vec{C}$ with edges in $S$ is neutral. A subgraph is balanced if its edge set is balanced (see $[1,4,17]$ for further details).

The following proposition gives necessary and sufficient conditions for a $\mathbb{T}$-gain graph to be balanced.

Proposition 2.1 ([17, Lemma 2.1]). Let $\Phi=(\Gamma, \gamma)$ be a $\mathbb{T}$-gain graph. Then the following are equivalent:

1. $\Phi$ is balanced.

2. $\Phi \sim(\Gamma, 1)$.

3. There exists a function $\theta: V(\Gamma) \rightarrow \mathbb{T}$ such that

$$
\theta\left(v_{i}\right)^{-1} \theta\left(v_{j}\right)=\gamma\left(e_{i j}\right) \quad \forall e_{i j} \in \vec{E}(\Gamma)
$$

By Proposition 2.1 (2), or [20, Theorem 2.8] we deduce the following corollary.

Corollary 2.2. A connected $\mathbb{T}$-gain graph $\Phi$ of order $n$ is balanced if and only if its least Laplacian eigenvalue $\mu_{n}(\Phi)$ is 0 .

The next proposition specializes $[18$, Lemma 2.2] to the case of $\mathbb{T}$-gain graphs.

Proposition 2.3. Let $\Phi_{1}=\left(\Gamma, \gamma_{1}\right)$ and $\Phi_{2}=\left(\Gamma, \gamma_{2}\right)$ be $\mathbb{T}$-gain graphs with the same underlying graph $\Gamma$. If for every cycle $C$ in $\Gamma$ there exists a directed cycle with base vertex $v$ such that $\gamma_{1}\left(\vec{C}_{v}\right)=\gamma_{2}\left(\vec{C}_{v}\right)$, then there exists a switching function $\zeta$ such that $\Phi_{2}=\Phi_{1}^{\zeta}$.

By Proposition 2.3, it follows that a gain graph $\Phi$ is balanced if and only if all its directed cycles are neutral.

Let $\Phi=(\Gamma, \gamma)$ be a complex unit gain graph, and let $X$ be a subset of $V(\Gamma)$. We write $\Phi[X]$ to denote the induced subgraph of $\Phi$ with vertex set $X$, and write $\Phi-X$ to denote $\Phi[V(\Gamma) \backslash X]$. As a consequence of the Cauchy's Interlacing Theorem for Hermitian matrices (see, for instance, [15, Theorem 4.3.17]), we arrive at the following result. 
Proposition 2.4. Let $\Phi=(\Gamma, \gamma)$ be a $\mathbb{T}$-gain graph of order $n$. For every $v \in V(\Gamma)$, the elements of $\operatorname{Spec}(A(\Phi))$ and $\operatorname{Spec}(A(\Phi-\{v\}))$ interlace as follows.

$$
\lambda_{1}(\Phi) \geqslant \lambda_{1}(\Phi-\{v\}) \geqslant \lambda_{2}(\Phi) \geqslant \lambda_{2}(\Phi-\{v\}) \geqslant \cdots \geqslant \lambda_{n-1}(\Phi-\{v\}) \geqslant \lambda_{n}(\Phi)
$$

From (2.1), it follows that the multiplicity of every eigenvalue $\lambda \in \operatorname{Spec}(A(\Phi))$ can change at most by 1 if some vertex is deleted. In view of this, a vertex $v$ is called downer, neutral, or Parter for $\lambda$ if the multiplicity of $\lambda$ decreases, remains the same, or increases, respectively. For some general results on the latter topic, we refer the reader to [19].

2.2. Star sets and star complements. Let $\Phi=(\Gamma, \gamma)$ be a complex unit gain graph, and let $m(\lambda)$ denote the multiplicity of the eigenvalue $\lambda \in \operatorname{Spec}(A(\Phi))$. A star set for $\lambda$ in $\Phi$ is a subset $X$ of $V(\Gamma)$ such that $\lambda \notin \operatorname{Spec}(A(\Phi-X))$ and $|X|=m(\lambda)$. The graph $\Phi-X$ is called a star complement of $\Phi$ with respect to $\lambda$.

In order to apply the star complement technique to complex unit gain graphs, we need to extend to Hermitian matrices some arguments given in [10, 12], where the authors only deal with real symmetric matrices.

Proposition 2.5. Let $\Phi=(\Gamma, \gamma)$ be a complex unit gain graph with $n$ vertices. For every eigenvalue $\lambda \in \operatorname{Spec}(A(\Phi))$, there exists a star set $X$ for $\lambda$.

Proof. Let $m(\lambda)$ be the multiplicity of a fixed $\lambda \in \operatorname{Spec}(A(\Phi))$. Since $\lambda I-A(\Phi)$ is a Hermitian matrix of rank $n-m(\lambda)$, one of its principal submatrices of order $n-m(\lambda)$, say $P$, is non-singular. Note that $P$ has the form $\lambda I-C$, where $C$ is a principal submatrix of $A(\Phi)$. This means that the vertices not corresponding to rows and columns in $C$ determine a star set for $\lambda$, and the remaining ones, that is, those corresponding to $C$, a star complement.

Here and throughout the rest of the paper, $N_{\Gamma}(v)$ (or simply $N(v)$ when it is clear which graph we are referring to) denotes the set of neighbors in a graph $\Gamma$ of a vertex $v \in V(\Gamma)$. The proof of the following theorem is constructive and resembles the one of Theorem 5.1.6 in [12].

Proposition 2.6. A connected complex unit gain graph $\Phi=(\Gamma, \gamma)$ has a connected star complement for each $\lambda \in \operatorname{Spec}(A(\Phi))$.

Proof. Since $\Gamma$ is connected, we can fix a labeling $\left\{v_{1}, \ldots, v_{n}\right\}$ for its vertices such that, for each $i \geqslant 2$, there exists a $v_{j} \in N\left(v_{i}\right)$ with $j<i$. Let $m(\lambda)$ be the multiplicity of a fixed $\lambda \in \operatorname{Spec}(A(\Phi))$, and let $c_{i}$ (resp. $c^{i}$ ) denote the $i$-th row (resp. the $i$-th column) of the matrix $\lambda I-A(\Phi)$. We now choose a subset of vertices $Y=\left\{v_{j_{1}}, \ldots, v_{j_{n-m(\lambda)}}\right\}$ according to the following procedure. We set $j_{1}=1$ and

$$
j_{h}=\min \left\{k>j_{h-1} \mid c^{k} \notin \operatorname{Span}\left(c^{j_{1}}, \ldots, c^{j_{h-1}}\right)\right\} \quad \text { for } 1<h \leqslant n-m(\lambda) .
$$

The columns $c^{j_{1}}, \ldots, c^{j_{n-m(\lambda)}}$ are linearly independent and generate the column space of $\lambda I-A(\Phi)$. Since such matrix is Hermitian, the rows $c_{j_{1}}, \ldots, c_{j_{n-m(\lambda)}}$ are linearly independent as well and generate the row space of $\lambda I-A(\Phi)$. Thus, the principal submatrix determined by the sequence $j_{1}<\cdots<j_{n-m(\lambda)}$ is nonsingular. This is equivalent to say that $\Phi[Y]$ is a star complement. We now show that $\Phi[Y]$ is connected by proving that each of its vertices (apart from the first one) is adjacent to a preceding one. For each $h>1$ let $k=\min \left\{i \mid v_{i} \in N\left(v_{j_{h}}\right)\right\}$. In our assumptions $k<j_{h}$. By definition of $k,-\gamma\left(e_{j_{h} k}\right)$ is the first non-zero element on the $j_{h}$-th row of $\lambda I-A$. This implies that $c^{k} \notin \operatorname{Span}\left(c^{1}, \ldots, c^{k-1}\right)$. Hence, $v_{k}$ belongs to $Y$.

Proposition 2.7. Let $\Phi=(\Gamma, \gamma)$ be a complex unit gain graph of order $n$, let $X=\left\{v_{i_{1}}, \ldots, v_{i_{m(\lambda)}}\right\}$ be a star set for $\lambda \in \operatorname{Spec}(A(\Phi))$, and let $X_{h}$ denote the set $\left\{v_{i_{1}}, \ldots, v_{i_{h}}\right\}$, for $1 \leqslant h \leqslant m(\lambda)$. The multiplicity of $\lambda$ for $A\left(\Phi-X_{h}\right)$ is $m(\lambda)-h$. 
Proof. By equation (2.1), the deletion of a vertex changes the multiplicity of every eigenvalue at most by 1 . The statement now comes from the fact that the multiplicity of $\lambda$ for the first and the last graph of the nested sequence

$$
\Phi-X=\Phi-X_{m(\lambda)} \subset \Phi-X_{m(\lambda)-1} \subset \cdots \subset \Phi-X_{2} \subset \Phi-X_{1} \subset \Phi,
$$

is 0 and $m$, respectively.

Corollary 2.8. Let $\Phi=(\Gamma, \gamma)$ be a complex unit gain graph, and let $X$ be a star set for $\lambda \in \operatorname{Spec}(A(\Phi))$. Denoted by $Y$ the set $V(\Gamma) \backslash X$, the multiplicity of $\lambda$ for the graph $\Phi[Y \cup\{v\}]$ is 1 for every $v \in X$.

Thanks to Corollary 2.8, we can extend to $\mathbb{T}$-gain graphs Theorem 7.3.1 in [10] without making use of projection maps and their properties.

Corollary 2.9. Let $\Phi=(\Gamma, \gamma)$ be a complex unit gain graph, $X$ be star set for $\lambda \neq 0$, and $\Phi[Y]$ be the corresponding star complement. Then, each vertex of $X$ has a neighbor in $Y$.

Proof. Assuming the contrary, a suitable vertex $v \in X$ would be isolated in $\Phi[Y \cup\{v\}]$; therefore, the multiplicity of $\lambda$ for both $\Phi[Y]$ and $\Phi[Y \cup\{v\}]$ would be 0 contradicting Corollary 2.8.

A basis for the eigenspace of $\lambda \in \operatorname{Spec}(A(\Phi))$ can be constructed as follows from the star complement $\Phi[Y]$ : for each $v \in X$ we consider a generator $\mathbf{y}_{\mathbf{v}}$ of the $\lambda$-eigenspace of $\Phi[Y \cup\{v\}]$ (its dimension is 1 by Corollary 2.8). A $\lambda$-eigenvector $\mathbf{x}_{\mathbf{v}}$ for $\Phi$ is obtained from $\mathbf{y}_{\mathbf{v}}$ by adding zero entries in correspondence of vertices in $X \backslash\{v\}$. By Proposition 2.7, the vertex $v \in X$ is a downer for $\lambda$; therefore, the $v$-component of $\mathbf{x}_{\mathbf{v}}$ is non-zero. It follows that the several $\mathbf{x}_{\mathbf{v}}$ 's for $v \in X$ are linearly independent and form a basis for $\mathcal{E}_{\Phi}(\lambda)$.

2.3. Line graphs associated with $\mathbb{T}$-gain graphs. Let $\Phi=(\Gamma, \gamma)$ be a $\mathbb{T}$-gain graph of order $n$ and size $m$. As in [17], the $n \times m$ complex matrix $\mathrm{H}(\Phi)=\left(\eta_{v e}\right)$ with entries in $\mathbb{T} \cup\{0\}$ is said to be an incidence matrix of $\Phi$ if

$$
\eta_{v_{i} e_{h}}= \begin{cases}-\eta_{v_{j} e_{h}} \gamma\left(e_{i j}\right) & \text { if the endpoints of } e_{h} \text { are precisely } v_{i} \text { and } v_{j}, \\ 0 & \text { otherwise. }\end{cases}
$$

In the case when $e_{h}$ joins $v_{i}$ and $v_{j}$, we also require that $\eta_{v_{i} e_{h}}$ is non-zero. We say 'an' incidence matrix, because by this definition $\mathrm{H}(\Phi)$ is unique only if $\Gamma$ is empty, that is, if it is of size 0 . If each column is multiplied by any element in $\mathbb{T}$, the resulting matrix is still an incidence matrix. Indeed, Proposition 2.10, whose proof is straightforward, says that all the other possible incidence matrices can be obtained from a fixed $\mathrm{H}(\Phi)$ in such a way.

Proposition 2.10. Let $\mathrm{H}(\Phi)=\left(\eta_{v e}\right)$ and $\mathrm{H}(\Phi)^{\prime}=\left(\eta_{v e}^{\prime}\right)$ be two incidence matrices both related to the $\mathbb{T}$-gain graph $\Phi=(\Gamma, \gamma)$. There exists an $m \times m$ diagonal matrix $S$ with entries in $\mathbb{T} \cup\{0\}$ such that $\mathrm{H}(\Phi)^{\prime}=\mathrm{H}(\Phi) S$ and $S^{*} S=I$.

By Proposition 2.10, for a fixed edge $e_{h} \in E(\Gamma)$ with endpoints $v_{i}$ and $v_{j}$, the possibilities for the non-zero elements on the corresponding column of $\mathrm{H}(\Phi)$ are

$$
\left(\eta_{v_{i} e_{h}}, \eta_{v_{j} e_{h}}\right)=\left(\mathrm{e}^{i \theta}, \mathrm{e}^{i(\theta+\pi)} \overline{\gamma\left(e_{i j}\right)}\right) \quad \text { for } 0 \leqslant \theta<2 \pi .
$$

In what follows, we denote by $\mathrm{H}$ a specific incidence matrix related to the $\mathbb{T}$-gain graph $\Phi=(\Gamma, \gamma)$. We next explain how $\mathrm{H}$ determines a $\mathbb{T}$-gain structure on the line graph $\mathcal{L}(\Gamma)$. It is well known that 
$V(\mathcal{L}(\Gamma))=E(\Gamma)$, and ef $\in E(\mathcal{L}(\Gamma))$ whenever $e$ and $f$ share an endpoint. We denote by $\mathcal{L}_{\mathrm{H}}(\Phi)$ the $\mathbb{T}$-gain graph $\left(\mathcal{L}(\Gamma), \gamma_{\mathrm{H}}^{\mathcal{L}}\right)$, where

$$
\gamma_{\mathrm{H}}^{\mathcal{L}}: e f \in \vec{E}(\mathcal{L}(\Gamma)) \longrightarrow \bar{\eta}_{w e} \eta_{w f} \in \mathbb{T}
$$

where $w$ is the endpoint shared by the edges $e$ and $f$. It is easy to verify that $\gamma_{\mathrm{H}}$ is a gain function. In fact,

$$
\gamma_{\mathrm{H}}^{\mathcal{L}}(f e)=\overline{\gamma_{\mathrm{H}}^{\mathcal{L}}(e f)}
$$

Given any Abelian group $\mathfrak{G}$, the gains for the line graph associated with a $\mathfrak{G}$-gain graph in [18] do not only depend on the chosen incidence matrix but also on the pick of a weak involution in $\mathfrak{G}$, that is, on an element $\mathfrak{s} \in \mathfrak{G}$ such that $\mathfrak{s}^{2}=1_{\mathfrak{G}}$. Our definition of $\mathcal{L}_{\mathrm{H}}(\Phi)$ is consistent with $\mathrm{N}$. Reff's for $\mathfrak{s}=1_{\mathfrak{G}}$ and $\mathfrak{G}=\mathbb{T}$.

THEOREM 2.11 ([18, Theorem 5.1]). Let $\mathrm{H}$ be one of the incidence matrices related to the $\mathbb{T}$-gain graph $\Phi=(\Gamma, \gamma)$. Then

$$
\mathrm{H}(\Phi)^{*} \mathrm{H}(\Phi)=2 I_{m}+A\left(\mathcal{L}_{H}(\Phi)\right)
$$

In a private communication to the authors, Tom Zaslavsky gave several arguments in favor of defining $\mathcal{L}_{\mathrm{H}}(\Phi)$ by picking a non-trivial weak involution in $\mathfrak{G}$ whenever it exists. Chosen $\mathfrak{s}=-1 \in \mathbb{T}$, Equation 2.3 should be replaced by [23, Theorem 5.1], and everything we say in Sections 3 and 4 on $\mathcal{E}_{\mathcal{L}_{H}(\Phi)}(-2)$ would hold for $\mathcal{E}_{\mathcal{L}_{H}(\Phi)}(2)$. Yet, we prefer to pick $\mathfrak{s}=1_{\mathbb{T}}$. In this way, our conclusions are more directly related to the classical results of Spectral Graph Theory collected in $[12,13]$. Moreover, when $\gamma(\vec{E}(\Gamma)) \subseteq\{-1,1\}$, that is, when the $\mathbb{T}$-gain graph $\Phi$ is actually a signed graph, and $\gamma_{\mathrm{H}}^{\mathcal{L}}$ is the gain function defined as in (2.2), we retrieve the same signature on $\mathcal{L}(\Phi)$ as assigned in [5, Section 1] and [6, Section 2].

We omit the proofs of Propositions 2.12, 2.13 and Theorem 2.14, since they are conceptually identical to those written down in [1] in the more restrictive context of $\mathbb{T}_{4}$-gain graphs.

Proposition 2.12 ([1, Proposition 5]). Let $\mathrm{H}$ and $\mathrm{H}^{\prime}$ be two of incidence matrices both associated with the same $\mathbb{T}$-gain graph $\Phi=(\Gamma, \gamma)$. Then $\mathcal{L}_{\mathrm{H}}(\Phi)$ and $\mathcal{L}_{\mathrm{H}^{\prime}}(\Phi)$ share the same adjacency spectrum. Moreover, if $S$ is the diagonal matrix such that $\mathrm{H}(\Phi)^{\prime}=\mathrm{H}(\Phi) S$, then

$$
A\left(\mathcal{L}_{\mathrm{H}^{\prime}}(\Phi)\right)=S^{*} A\left(\mathcal{L}_{\mathrm{H}}(\Phi)\right) S .
$$

Proposition 2.13 ([1, Proposition 6 and its proof $]$ ). Line graphs of switching equivalent $\mathbb{T}$-gain graphs $\Phi_{1}=\left(\Gamma, \gamma_{1}\right)$ and $\Phi_{2}=\left(\Gamma, \gamma_{2}\right)$ are switching equivalent. Moreover, if $\zeta: V(\Gamma) \rightarrow \mathbb{T}$ is the switching function such that $\Phi_{2}=\Phi_{1}^{\zeta}$, and $\mathrm{H}_{1}$ is an incidence matrix for $\Phi_{1}$, then $D(\zeta)^{-1} \mathrm{H}_{1}$ is an incidence matrix for $\Phi_{2}$, and

$$
\mathcal{L}_{\mathrm{H}_{1}}\left(\Phi_{1}\right)=\mathcal{L}_{D(\zeta)^{-1} \mathrm{H}_{1}}\left(\Phi_{2}\right)
$$

The final result of this section concerns the mutual interrelationships between the Laplacian polynomial of a $\mathbb{T}$-gain graph $\Phi$ and the adjacency polynomial of its line graphs. Proposition 2.12 allows us to drop the incidence matrix out of notations in the statements.

TheOREm 2.14 ([1, Theorem 4]). Let $\Gamma$ be a graph of order $n$ and size $m$, and $\Phi$ a $\mathbb{T}$-gain graph having $\Gamma$ as underlying graph. Then

$$
\phi(\mathcal{L}(\Phi), x)=(x+2)^{m-n} \psi(\Phi, x+2)
$$

Since the Laplacian eigenvalues of a complex unit graph are all nonnegative, from (2.4) it immediately follows that no eigenvalue in $\operatorname{Spec}(A(\mathcal{L}(\Phi)))$ is less than -2 . 
Electronic Journal of Linear Algebra, ISSN 1081-3810

3. An eigenbasis for -2 in complex unit line graphs. Let $\Phi=(\Gamma, \gamma)$ be a complex unit gain graph, and let $\mathcal{L}(\Phi)=\left(\mathcal{L}(\Gamma), \gamma^{\mathcal{L}}\right)$ be the associated line graph arising from a fixed incidence matrix $\mathrm{H}$ of $\Phi$. The first theorem of this section identifies the structural conditions on $\Phi$ ensuring the presence of -2 in $\operatorname{Spec}(A(\mathcal{L}(\Phi)))$.

THEOREM 3.1. Let $\Phi=(\Gamma, \gamma)$ be a connected complex unit gain graph of order $n$ and size $m$, and $\overrightarrow{\mathcal{C}}(\Gamma)$ be the set of directed cycles in $\Gamma$. Then,

$$
(-1)^{m} \phi(\mathcal{L}(\Phi),-2)= \begin{cases}m+1 & \text { if } \Gamma \text { is a tree, } \\ 2-2 \cos \theta & \text { if }(\Gamma, \gamma) \text { is unbalanced unicyclic and } \gamma(\vec{C})=\mathrm{e}^{i \theta} \text { for a } \vec{C} \in \overrightarrow{\mathcal{C}}(\Gamma), \\ 0 & \text { otherwise. }\end{cases}
$$

Proof. If $\Gamma$ is a tree, then $\Phi$ is balanced. Therefore, $\Phi \sim(\Gamma, 1)$, and by Proposition 2.13, we get $\phi(\mathcal{L}(\Phi))=$ $\phi(\mathcal{L}(\Gamma, 1))$. The equality $(-1)^{m} \phi(\mathcal{L}(\Phi),-2)=m+1$ now comes from [12, Lemma 7.5.2(i)] or [7, Lemma 3.8]. In fact, $(\Gamma, 1)$ can be regarded as an unsigned graph.

Let now $\Gamma$ be unicyclic. Equation (2.4) specializes to

$$
\phi(\mathcal{L}(\Phi),-2)=\psi(\Phi, 0)=\operatorname{det}(L(\Phi)) .
$$

Since $\Gamma$ is unicyclic, the directed cycles in $\overrightarrow{\mathcal{C}}(\Gamma)$ have just two possible gains. Such gains are complex conjugate, say $\mathrm{e}^{i \theta}$ and $\mathrm{e}^{-i \theta}$. Fixed any $\vec{C} \in \overrightarrow{\mathcal{C}}(\Gamma)$, from [20, Lemmas 2.2 and 2.4] we deduce

$$
\operatorname{det}(L(\Phi))=\operatorname{det}(\vec{C})=|1-\gamma(\vec{C})|^{2}=(1-\gamma(\vec{C}))\left(1-\gamma^{-1}(\vec{C})\right)=2-2 \cos \theta .
$$

Finally, if $\Gamma$ is neither a tree nor a unicyclic graph, then $m>n$, and $(-1)^{m} \phi(\mathcal{L}(\Phi),-2)=0$ by Theorem 2.14.

Corollary 3.2. Let $\Phi$ be a connected complex unit gain graph. The least eigenvalue of $\mathcal{L}(\Phi)$ is -2 if and only if $\Phi$ contains as a complex unit subgraph at least one balanced cycle or two unbalanced cycles.

In what follows, we attempt to stick as close as possible to the way of arguing of [5, Section 3], where an eigenbasis for -2 in signed lined graphs is detected.

Unless told otherwise, we assume the underlying graph $\Gamma$ of the complex unit gain graph $\Phi$ (and therefore $\mathcal{L}(\Gamma))$ is connected, and -2 belongs to $\operatorname{Spec}(A(\mathcal{L}(\Phi))$ with multiplicity $k>0$. We now use the ideas explained in the last paragraph of Section 2.2 to find an eigenbasis for $\lambda=-2$. Such basis will arise from a connected star complement in $\mathcal{L}(\Phi)$ related to -2 .

By Proposition 2.6, $\mathcal{L}(\Phi)$ has a connected induced subgraph which is a star complement with respect to $\lambda=-2$. The corresponding edges in $\Phi$ induce the 'line star complement', which is also connected apart from isolated vertices, if any. In the spirit of $[5,11]$, every line star complement in $\Phi$ with respect to -2 is also called a foundation. Henceforth, we assume $\Psi=\left(\Lambda, \gamma_{\mid \vec{E}(\Lambda)}\right)$ is a fixed foundation. Since the isolated vertices do not affect $\mathcal{L}(\Psi) \subset \mathcal{L}(\Phi)$, it is not restrictive to assume $\Psi$ is connected. If this is the case, by Theorem 3.1, $\Psi$ is either a tree or an unbalanced unicyclic graph.

As discussed in Section 2.2, the procedure to obtain a (-2)-eigenbasis of $\mathcal{L}(\Phi)$ consists of enriching the induced subgraph $\mathcal{L}(\Psi)$ by a vertex in $V(\mathcal{L}(\Gamma)) \backslash V(\mathcal{L}(\Lambda)$ ), or equivalently in adding an edge $e \in E(\Gamma) \backslash E(\Lambda)$ to $\Lambda$. We set $\Psi_{e}:=\left(\Lambda_{e}, \gamma_{\mid \vec{E}\left(\Lambda_{e}\right)}\right)$, where $V\left(\Lambda_{e}\right)=V(\Lambda)$ and $E\left(\Lambda_{e}\right)=E(\Lambda) \cup\{e\}$. Let now $\mathbf{x}_{\mathbf{e}}$ be a $(-2)$ eigenvector of $\mathcal{L}\left(\Psi_{e}\right)$. Each of its coordinates is labeled by a suitable edge in $\Psi_{e}$. By Corollary 2.8 applied 
to $\mathcal{L}\left(\Psi_{e}\right)$, the $(-2)$-eigenspace of its adjacency matrix is one-dimensional. Thus, every (-2)-eigenvector $\mathbf{v}$ of $\mathcal{L}\left(\Psi_{e}\right)$ is proportional to $\mathbf{x}_{\mathbf{e}}$, in particular $\mathbf{v}$ shares with $\mathbf{x}_{\mathbf{e}}$ the same non-zero versus zero pattern.

In view of the latter observation, we can distinguish two types of edges in $\Psi_{e}$. We say that an edge is heavy (resp. light) when the corresponding entry in $\mathbf{x}_{\mathbf{e}}$ is non-zero (resp. zero). The unique subgraph $\Theta_{e}$ of $\Psi_{e}$ induced by its heavy edges will be called the core of $\Psi_{e}$. Throughout the rest of this section, the words 'downer', 'neutral', and 'Parter' will always be used to qualify the vertices of a certain line graph with respect to the eigenvalue -2 .

Proposition 3.3. The vertices in $\mathcal{L}\left(\Psi_{e}\right)$ corresponding to edges of $\Theta_{e}$ are downers, the remaining ones are neutrals.

Proof. Since $\lambda=-2$ is the least eigenvalue for $A\left(\mathcal{L}\left(\Psi_{e}\right)\right)$, from (2.1) it follows that the graph $\mathcal{L}\left(\Psi_{e}\right)$ has no Parter vertices. It is routine to check that vertices corresponding to light edges are neutral. Now, assume by contradiction that an edge $f$ of $\Theta_{e}$ corresponds to a neutral vertex for $\mathcal{L}\left(\Psi_{e}\right)$. There would exist a -2-eigenvector $\mathbf{y}_{\mathbf{e}}$ for $A\left(\mathcal{L}\left(\Psi_{e}\right)-\{f\}\right)$, and a -2-eigenvector $\mathbf{y}_{\mathbf{e}}^{\prime}$ for $A\left(\mathcal{L}\left(\Psi_{e}\right)\right)$ obtained from $\mathbf{y}_{\mathbf{e}}$ by inserting a 0-entry in correspondence of $f \in V\left(\mathcal{L}\left(\Psi_{e}\right)\right)$. Clearly $\mathbf{y}_{\mathbf{e}}^{\prime}$ would not be proportional to $\mathbf{x}_{\mathbf{e}}$, against the one-dimensionality of the -2-eigenspace for $A\left(\mathcal{L}\left(\Psi_{e}\right)\right)$. Hence, such a 'downer' $f$ in $\Theta_{e}$ does not exist.

The next proposition collects some properties of the core $\Theta_{e}$.

Proposition 3.4. Let $\Theta_{e}$ be the core of the graph $\Psi_{e}$ built from a connected foundation $\Psi=\left(\Lambda, \gamma_{\mid \vec{E}(\Lambda)}\right)$ of a connected complex unit graph $\Phi=(\Gamma, \gamma)$ and an edge $e \in E(\Gamma) \backslash E(\Lambda)$. The following properties hold.

(i) The edge e belongs to $\Theta_{e}$.

(ii) $\Theta_{e}$ is connected.

(iii) No edge in $\Theta_{e}$ is pendant.

(iv) The edge e belongs to some cycle of $\Theta_{e}$.

Proof. Since the graph $\Psi$ is a foundation, the vertex in $\mathcal{L}\left(\Psi_{e}\right)$ corresponding to $e$ is a downer. Therefore, Part (i) comes from Proposition 3.3.

Let $X_{e}$ be the connected component of $\Theta_{e}$ containing $e$. Note that $-2 \in \operatorname{Spec}\left(A\left(\mathcal{L}\left(X_{e}\right)\right)\right)$, otherwise $e$ would not be a downer for $\mathcal{L}\left(\Psi_{e}\right)$. The multiplicity of -2 in $\operatorname{Spec}\left(A\left(\mathcal{L}\left(X_{e}\right)\right)\right)$ is necessarily one, being one in $\operatorname{Spec}\left(A\left(\mathcal{L}\left(\Psi_{e}\right)\right)\right)$. This implies that every edge in $\Theta_{e} \backslash X_{e}$, if existing, would be neutral against Proposition 3.3 . It follows that $X_{e}=\Theta_{e}$, proving Part (ii).

Since $\Theta_{e}$ is connected and -2 is an eigenvalue for $A\left(\mathcal{L}\left(\Theta_{e}\right)\right.$ (of multiplicity one), Corollary 3.2 implies that $\Theta_{e}$ contains as complex unit subgraph at least a balanced cycle or at least two unbalanced cycles. For each pendant edge $f$, the same thing would be true for the connected complex unit graph $\Theta_{e}-\{f\}$. Again by Corollary 3.2, we would infer that -2 is an eigenvalue for $A\left(\mathcal{L}\left(\Theta_{e}-\{f\}\right)\right)$, and the edge $f$ would be neutral against Proposition 3.3. Hence, no pendant edges exist as stated in Part (iii).

Part (iv) is proved by contradiction. Since $\Theta_{e}$ does not contain pendant edges, if $e$ does not belong to a cycle, then it should be a bridge. By Part (i), we know that $-2 \notin \operatorname{Spec}\left(A\left(\mathcal{L}\left(\Theta_{e}-\{e\}\right)\right)\right)$. By Corollary 3.2, no component of $\Theta_{e}-\{e\}$ contains a balanced cycle or two unbalanced cycles. This implies that $e$ would belong to a path connecting two unbalanced cycles. Recall now that, in our hypotheses, the graph foundation $\Psi$ is connected, and $\Theta_{e}-\{e\} \subset \Psi$. Hence, we can find in $\Psi$ two unbalanced cycles joined by a path against Corollary 3.2 . 
From Proposition 3.4, and by Corollary 3.2 applied to $\Psi$, we conclude that the core $\Theta_{e}$ is either a balanced cycle, or a dumbbell whose two cycles are both unbalanced, or an $\infty$-graph with two unbalanced cycles. Recall that a dumbbell is a graph consisting of two disjoint cycles joined by a non-trivial path, whereas an $\infty$-graph consists of two cycles with just one vertex in common.

So the problem of constructing (-2)-eigenvectors in complex unit line graphs is reduced to finding those eigenvectors arising from the cores described above.

TheOREm 3.5. Let the core $\Theta_{e}=\left(C, \gamma_{\mid \vec{E}(C)}\right)$ be a balanced cycle. After labeling the $q \geqslant 3$ vertices of $C$ and its edges as in Fig. 1, a generator $\mathbf{a}=\left(a_{0}, a_{1}, \ldots, a_{q-1}\right)^{\top}$ of the-2-eigenspace of $A\left(\mathcal{L}\left(\Theta_{e}\right)\right)$ is given by the formula

$$
a_{i}=(-1)^{i}\left[\prod_{s=1}^{i} \overline{\nu(s)}\right] a_{0} \quad \text { for } 1 \leqslant i \leqslant q-1 \quad \text { and } \quad a_{0} \neq 0,
$$

where the component $a_{i}$ corresponds to the edge $e_{i}$, and

$$
\nu(i)=\gamma^{\mathcal{L}}\left(e_{i-1} e_{i}\right)=\bar{\eta}_{i e_{i-1}} \eta_{i e_{i}} \in \mathbb{T} \quad \text { for } 1 \leqslant i \leqslant q-1 .
$$

Moreover, the vector a can be extended to a (-2)-eigenvector of $A(\mathcal{L}(\Phi))$ by inserting zeros at the remaining entries.

Proof. Vertices and edges of the cycle $C$ are labeled as follows:

$$
V(C)=\left\{v_{0}, \ldots, v_{q-1}\right\}, \quad \text { and } \quad E(C)=\left\{e_{i}=v_{i} v_{i+1} \mid 0 \leqslant i \leqslant q-2\right\} \cup\left\{e_{q-1}=v_{q-1} v_{0}\right\} .
$$

Let $\mathbf{x}=\left(x_{0}, x_{1}, \ldots, x_{q-1}\right)^{\top}$ be a $(-2)$-eigenvector of $\mathcal{L}\left(\Theta_{e}\right)$. By using (3.5), the equation $A\left(\mathcal{L}\left(\Theta_{e}\right)\right) \mathbf{x}=$ $-2 \mathrm{x}$ yields

$$
\begin{aligned}
-2 x_{0} & =\overline{\nu(0)} x_{q-1}+\nu(1) x_{1}, \\
-2 x_{i} & =\overline{\nu(i)} x_{i-1}+\nu(i+1) x_{i+1} \quad \text { for } 0<i<q-1, \\
-2 x_{q-1} & =\overline{\nu(q-1)} x_{q-2}+\nu(0) x_{0},
\end{aligned}
$$

where we set $\nu(0)=\gamma^{\mathcal{L}}\left(e_{q-1} e_{0}\right)=\bar{\eta}_{0 e_{q-1}} \eta_{0 e_{0}}$.

Now we fix a non-zero complex number $a_{0}$ and choose as a 'guessing solution' the vector

$$
\mathbf{a}=\left(a_{0}, a_{1}, \ldots, a_{q-1}\right)^{\top},
$$

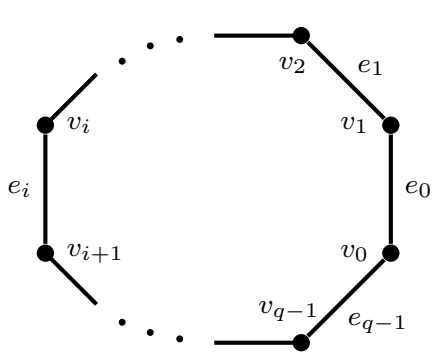

FiguRE 1. Vertex and edge labeling for the core $\Theta_{e}$ being a cycle. 
with

$$
a_{i+1}=-\overline{\nu(i+1)} a_{i} \quad \text { or, equivalently, } a_{i}=-\nu(i+1) a_{i+1} \quad \text { for } 0 \leqslant i \leqslant q-2 .
$$

Note that $\mathbf{a}$ is a vector of the type described in the statement. Its components satisfy the second equation in (3.6). In order to realize that a satisfies the 'boundary conditions' as well, that is, the first and the third equation in (3.6), we observe that the conditions

$$
-2 a_{0}=\overline{\nu(0)} a_{q-1}+\nu(1) a_{1} \quad \text { and } \quad-2 a_{q-1}=\overline{\nu(q-1)} a_{q-2}+\nu(0) a_{0}
$$

are both equivalent to

$$
\prod_{s=0}^{q-1} \overline{\nu(s)}=(-1)^{q}
$$

which actually holds, since in general

$$
\prod_{s=0}^{q-1} \overline{\nu(s)}=(-1)^{q} \overline{\gamma\left(\vec{C}_{0}\right)}, \quad \text { where } \vec{C}_{0}=e_{01} e_{12} \cdots e_{(q-1) 0}
$$

and in our hypotheses $\gamma\left(\vec{C}_{0}\right)=1$.

As Tom Zaslavsky privately pointed out to the authors, for $1 \leqslant i \leqslant q-1$, the numbers $\left[\prod_{s=1}^{i} \overline{\nu(s)}\right]$ appearing in the statement of Theorem 3.5 have an intriguing geometric meaning: they compute the gains of the several paths $P_{i 0}$ 's in $\mathcal{L}(\Phi)$ where $P_{i 0}:=e_{i} e_{i-1} \cdots e_{0}$.

We now fix some notation to investigate the cases when the underlying graph of $\Theta_{e}$ consists of two cycles $C^{\prime}$ and $C^{\prime \prime}$ (of length $q^{\prime}$ and $q^{\prime \prime}$, respectively) joined by a path $P$ of length $p \geqslant 0$. In literature, this bicyclic graph is often denoted by $B\left(q^{\prime}, p, q^{\prime \prime}\right)$ (see, for instance, $[8,10]$ ). We label vertices and edges of $\Theta_{e}$ as follows:

$$
\begin{gathered}
V\left(C^{\prime}\right)=\left\{v_{0}^{\prime}, \ldots, v_{q^{\prime}-1}^{\prime}\right\}, \quad V\left(C^{\prime \prime}\right)=\left\{v_{0}^{\prime \prime}, \ldots, v_{q^{\prime \prime}-1}^{\prime \prime}\right\}, \\
E\left(C^{\prime}\right)=\left\{e_{i}^{\prime}=v_{i}^{\prime} v_{i+1}^{\prime} \mid 0 \leqslant i \leqslant q^{\prime}-2\right\} \cup\left\{e_{q^{\prime}-1}^{\prime}=v_{q^{\prime}-1}^{\prime} v_{0}^{\prime}\right\}, \\
E\left(C^{\prime \prime}\right)=\left\{e_{i}^{\prime \prime}=v_{i}^{\prime \prime} v_{i+1}^{\prime \prime} \mid 0 \leqslant i \leqslant q^{\prime \prime}-2\right\} \cup\left\{e_{q^{\prime \prime}-1}^{\prime \prime}=v_{q^{\prime \prime}-1}^{\prime \prime} v_{0}^{\prime \prime}\right\} .
\end{gathered}
$$

If $P$ is non-trivial, that is, if its length is $p>0$, we assume that

$$
V(P)=\left\{w_{0}, \ldots, w_{p}\right\}, \quad E(P)=\left\{f_{i}=w_{i} w_{i+1} \mid 0 \leqslant i \leqslant p-1\right\}
$$

and its end-vertices $w_{0}$ and $w_{p}$ are, respectively, identified with vertices $v_{0}^{\prime} \in V\left(C^{\prime}\right)$ and $v_{0}^{\prime \prime} \in V\left(C^{\prime \prime}\right)($ see Figs. 2 and 3).

Let $\mathbf{x}$ be a -2-eigenvector for $A\left(\mathcal{L}\left(\Theta_{e}\right)\right)$. For convenience, we split its ordered set of components into three (resp. two) parts if $p>0$ (resp. $p=0$ ), each corresponding to its constituents $C^{\prime}, P$ (if non-trivial), and $C^{\prime \prime}$. Namely, we write $\mathbf{x}=\mathbf{a}^{\prime} \dot{+} \mathbf{b} \dot{+} \mathbf{a}^{\prime \prime}$ where $\mathbf{a}^{\prime}=\left(a_{0}^{\prime}, a_{1}^{\prime}, \ldots, a_{q^{\prime}-1}^{\prime}\right)^{\top}, \mathbf{b}=\left(b_{0}, b_{1}, \ldots, b_{p-1}\right)^{\top}$, and $\mathbf{a}^{\prime \prime}=\left(a_{0}^{\prime \prime}, a_{1}^{\prime \prime}, \ldots, a_{q^{\prime \prime}-1}^{\prime \prime}\right)^{\top}$, and the components $a_{i}^{\prime}, b_{i}$, and $a_{i}^{\prime \prime}$ respectively correspond to the edges $e_{i}^{\prime}, f_{i}$, and $e_{i}^{\prime \prime}$. In the statements of Theorems 3.6 and 3.7, the following two directed cycles

$$
\vec{C}_{0}^{\prime}=e_{01}^{\prime} e_{12}^{\prime} \cdots e_{\left(q^{\prime}-1\right) 0}^{\prime} \quad \text { and } \quad \vec{C}_{0}^{\prime \prime}=e_{01}^{\prime \prime} e_{12}^{\prime \prime} \cdots e_{\left(q^{\prime \prime}-1\right) 0}^{\prime \prime}
$$

where $e_{i j}^{\prime}=v_{i}^{\prime} v_{j}^{\prime}$ and $e_{i j}^{\prime \prime}=v_{i}^{\prime \prime} v_{j}^{\prime \prime}$, play an important role. 


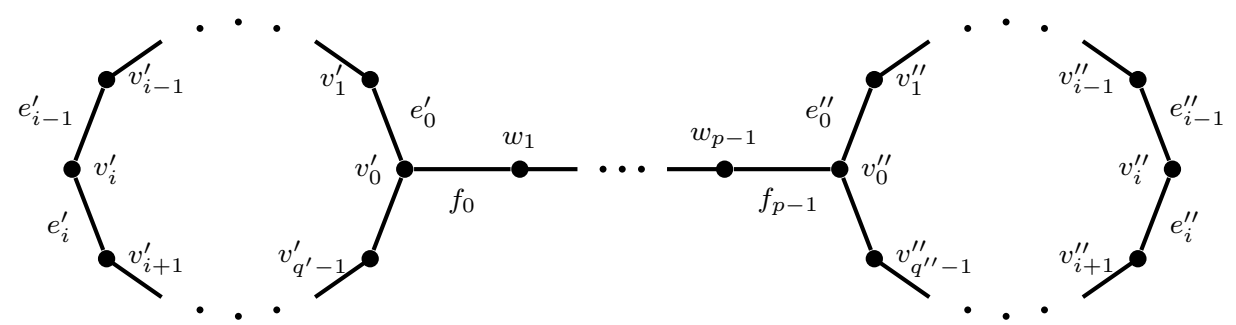

Figure 2. Vertex and edge labeling for the core $\Theta_{e}$ being a dumbbell.

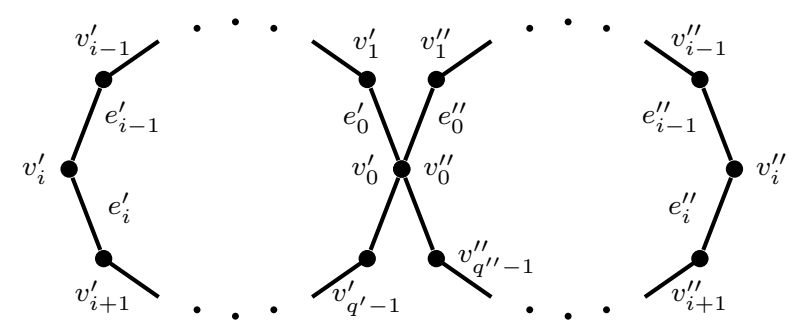

Figure 3. Vertex and edge labeling for the core $\Theta_{e}$ being a $\infty$-graph.

TheOREM 3.6. Let the core $\Theta_{e}=\left(B\left(q^{\prime}, p, q^{\prime \prime}\right), \gamma_{\mid \vec{E}\left(B\left(q^{\prime}, p, q^{\prime \prime}\right)\right)}\right)$ be a complex unit dumbbell with two unbalanced cycles. Under the above notation (see also Fig. 2), for each non-zero complex number $b_{0}$, a generator $\mathbf{a}^{\prime} \dot{+} \mathbf{b} \dot{+} \mathbf{a}^{\prime \prime}$ of the -2-eigenspace of $A\left(\mathcal{L}\left(\Theta_{e}\right)\right)$ is given by the formula

$$
a_{0}^{\prime}=-\left(1-\overline{\gamma\left(\overrightarrow{C_{0}^{\prime}}\right)}\right)^{-1} \gamma^{\mathcal{L}}\left(e_{0}^{\prime} f_{0}\right) b_{0}, \quad a_{0}^{\prime \prime}=-\left(1-\overline{\gamma\left(\overrightarrow{C_{0}^{\prime \prime}}\right)}\right)^{-1} \gamma^{\mathcal{L}}\left(e_{0}^{\prime \prime} f_{p-1}\right) b_{p-1}
$$

and

$$
\begin{aligned}
& a_{i}^{\prime}=(-1)^{i}\left[\prod_{s=1}^{i} \overline{\nu^{\prime}(s)}\right] a_{0}^{\prime} \quad \text { for } 1 \leqslant i \leqslant q^{\prime}-1, \\
& b_{i}=(-1)^{i}\left[\prod_{s=1}^{i} \overline{\nu(s)}\right] b_{0} \quad \text { for } 1 \leqslant i \leqslant p-1 \quad \text { and } \quad b_{0} \neq 0, \\
& a_{i}^{\prime \prime}=(-1)^{i}\left[\prod_{s=1}^{i} \overline{\nu^{\prime \prime}(s)}\right] a_{0}^{\prime \prime} \quad \text { for } 1 \leqslant i \leqslant q^{\prime \prime}-1,
\end{aligned}
$$

where

$$
\begin{array}{cc}
\nu^{\prime}(i)=\gamma^{\mathcal{L}}\left(e_{i-1}^{\prime} e_{i}^{\prime}\right)=\bar{\eta}_{i e_{i-1}^{\prime}} \eta_{i e_{i}^{\prime}} \in \mathbb{T} & \text { for } 1 \leqslant i \leqslant q^{\prime}-1, \\
\nu(i)=\gamma^{\mathcal{L}}\left(f_{i-1} f_{i}\right)=\bar{\eta}_{i f_{i-1}} \eta_{i f_{i}} \in \mathbb{T} & \text { for } 1 \leqslant i \leqslant p-1, \\
\nu^{\prime \prime}(i)=\gamma^{\mathcal{L}}\left(e_{i-1}^{\prime \prime} e_{i}^{\prime \prime}\right)=\bar{\eta}_{i e_{i-1}^{\prime \prime}} \eta_{i e_{i}^{\prime \prime}} \in \mathbb{T} & \text { for } 1 \leqslant i \leqslant q^{\prime \prime}-1 .
\end{array}
$$

Moreover, $\mathbf{a}^{\prime} \dot{+} \mathbf{b} \dot{+} \mathbf{a}^{\prime \prime}$ can be extended to a (-2)-eigenvector of $A(\mathcal{L}(\Phi))$ by putting zeros at all other entries. 
Electronic Journal of Linear Algebra, ISSN 1081-3810

A publication of the International Linear Algebra Society

Volume 37, pp. 14-30, January 2021

Proof. We start by setting

$$
\nu^{\prime}(0)=\gamma^{\mathcal{L}}\left(e_{q^{\prime}-1}^{\prime} e_{0}^{\prime}\right)=\bar{\eta}_{v_{0}^{\prime} e_{q^{\prime}-1}^{\prime}} \eta_{v_{0}^{\prime} e_{0}^{\prime}} \quad \text { and } \quad \nu^{\prime \prime}(0)=\gamma^{\mathcal{L}}\left(e_{q^{\prime \prime}-1}^{\prime \prime} e_{0}^{\prime \prime}\right)=\bar{\eta}_{v_{0}^{\prime \prime} e_{q^{\prime \prime}-1}^{\prime \prime}} \eta_{v_{0}^{\prime \prime} e_{0}^{\prime \prime}}
$$

By definition, we get

$$
\prod_{s=0}^{q^{\prime}-1} \overline{\nu^{\prime}(s)}=(-1)^{q^{\prime}} \overline{\gamma\left(\vec{C}_{0}^{\prime}\right)} \quad \text { and } \quad \prod_{s=0}^{q^{\prime \prime}-1} \overline{\nu^{\prime \prime}(s)}=(-1)^{q^{\prime \prime}} \overline{\gamma\left(\vec{C}_{0}^{\prime \prime}\right)}
$$

We have to check that $A\left(\mathcal{L}\left(\Theta_{e}\right)\right)\left(\mathbf{a}^{\prime} \dot{+} \mathbf{b} \dot{+} \mathbf{a}^{\prime \prime}\right)=-2\left(\mathbf{a}^{\prime} \dot{+} \mathbf{b} \dot{+} \mathbf{a}^{\prime \prime}\right)$. The eigenvalue equations at vertices of degree 2 in $\mathcal{L}\left(\Theta_{e}\right)$ resemble the middle equation in (3.6), and it is not hard to show that they actually hold by looking at (3.8)-(3.10). The non-trivial checks involve the vertices in correspondence of the edges $e_{0}^{\prime}$, $e_{q^{\prime}-1}^{\prime}$, and $f_{0}$ (all incident to $v_{0}^{\prime}$ ), and $e_{0}^{\prime \prime}, e_{q^{\prime \prime}-1}^{\prime \prime}$, and $f_{p-1}$ (all incident to $v_{0}^{\prime \prime}$ ). By virtue of symmetry, we provide the verification just for $e_{0}^{\prime}$ and $f_{0}$.

Consider first the edge $e_{0}^{\prime}$. We have to check the equality

$$
(-2) a_{0}^{\prime}=\nu^{\prime}(1) a_{1}^{\prime}+\overline{\nu^{\prime}(0)} a_{q^{\prime}-1}^{\prime}+\gamma^{\mathcal{L}}\left(e_{0}^{\prime} f_{0}\right) b_{0}
$$

When you make the substitutions

$$
a_{1}^{\prime}=-\overline{\nu^{\prime}(1)} a_{0}^{\prime}, \quad a_{q^{\prime}-1}^{\prime}=(-1)^{q^{\prime}-1}\left[\prod_{s=1}^{q^{\prime}-1} \overline{\nu^{\prime}(s)}\right] a_{0}^{\prime} \quad \text { and } \quad b_{0}=-\left(1-\overline{\gamma\left(\overrightarrow{C_{0}^{\prime}}\right)}\right) \gamma^{\mathcal{L}}\left(f_{0} e_{0}^{\prime}\right)
$$

coming from (3.7) and (3.8), Equality 3.16 becomes in fact equivalent to the first equation of (3.15).

Consider secondly the edge $f_{0}$. We have to check the equality

$$
-2 b_{0}=\gamma^{\mathcal{L}}\left(f_{0} e_{0}^{\prime}\right) a_{0}^{\prime}+\gamma^{\mathcal{L}}\left(f_{0} e_{q-1}^{\prime}\right) a_{q^{\prime}-1}^{\prime}+ \begin{cases}\gamma^{\mathcal{L}}\left(f_{0} e_{0}^{\prime \prime}\right) a_{0}^{\prime \prime}+\gamma^{\mathcal{L}}\left(f_{0} e_{q^{\prime \prime}-1}^{\prime \prime}\right) a_{q^{\prime \prime}-1}^{\prime \prime} & \text { if } p=1 \\ \nu(1) b_{1} & \text { if } p>1\end{cases}
$$

To this aim, we observe that

$$
\gamma^{\mathcal{L}}\left(f_{0} e_{0}^{\prime}\right) a_{0}^{\prime}=-\left(1-\overline{\gamma\left(\overrightarrow{C_{0}^{\prime}}\right)}\right)^{-1} b_{0} \quad \text { by }(3.7)
$$

and

$$
\begin{aligned}
& \gamma^{\mathcal{L}}\left(f_{0} e_{q-1}^{\prime}\right) a_{q^{\prime}-1}^{\prime}=\bar{\eta}_{v_{0}^{\prime} f_{0}} \eta_{v_{0}^{\prime} e_{q^{\prime}-1}^{\prime}} \cdot(-1)^{q^{\prime}-1}\left[\prod_{s=1}^{q^{\prime}-1} \overline{\nu^{\prime}(s)}\right] a_{0}^{\prime} \quad \text { by (2.2) and (3.8), } \\
& =\bar{\eta}_{v_{0}^{\prime} f_{0}} \eta_{v_{0}^{\prime} e_{q^{\prime}-1}^{\prime}} \nu^{\prime}(0) \cdot(-1)^{q^{\prime}-1}\left[\prod_{s=0}^{q^{\prime}-1} \overline{\nu^{\prime}(s)}\right] a_{0}^{\prime} \\
& =\bar{\eta}_{v_{0}^{\prime} f_{0}} \eta_{v_{0}^{\prime} e_{0}^{\prime}}(-1)^{q^{\prime}-1}(-1)^{q^{\prime}} \overline{\gamma\left(\vec{C}_{0}^{\prime}\right)} a_{0}^{\prime} \quad \text { by (3.14) and (3.15), } \\
& =\gamma^{\mathcal{L}}\left(f_{0} e_{0}^{\prime}\right) \gamma^{\mathcal{L}}\left(e_{0}^{\prime} f_{0}\right) \overline{\gamma\left(\vec{C}_{0}^{\prime}\right)}\left(1-\overline{\gamma\left(\overrightarrow{C_{0}^{\prime}}\right)}\right)^{-1} b_{0} \quad \text { by }(2.2) \text { and }(3.7) \text {. } \\
& =\overline{\gamma\left(\vec{C}_{0}^{\prime}\right)}\left(1-\overrightarrow{\gamma\left(\overrightarrow{C_{0}^{\prime}}\right)}\right)^{-1} b_{0}
\end{aligned}
$$


Hence, (3.17) is equivalent to

$$
-b_{0}= \begin{cases}\gamma^{\mathcal{L}}\left(f_{0} e_{0}^{\prime \prime}\right) a_{0}^{\prime \prime}+\gamma^{\mathcal{L}}\left(f_{0} e_{q^{\prime \prime}-1}^{\prime \prime}\right) a_{q^{\prime \prime}-1}^{\prime \prime} & \text { if } p=1, \\ \nu(1) b_{1} & \text { if } p>1 .\end{cases}
$$

For $p>1,(3.18)$ follows from $b_{1}=-\overline{\nu(1)} b_{0}$, which is (3.9) specialized to the case $i=1$. For $p=1$, note that

$$
\gamma^{\mathcal{L}}\left(f_{0} e_{0}^{\prime \prime}\right) a_{0}^{\prime \prime}=-\left(1-\overrightarrow{\gamma\left(\overrightarrow{C_{0}^{\prime \prime}}\right)}\right)^{-1} b_{0} \quad \text { by }(3.7),
$$

and, arguing as above,

$$
\begin{aligned}
\gamma^{\mathcal{L}}\left(f_{0} e_{q^{\prime \prime}-1}^{\prime \prime}\right) a_{q^{\prime \prime}-1}^{\prime \prime} & =\gamma^{\mathcal{L}}\left(f_{0} e_{q^{\prime \prime}-1}^{\prime \prime}\right)(-1)^{q^{\prime \prime}}-1\left[\prod_{s=1}^{q^{\prime \prime}-1} \overline{\nu^{\prime \prime}(s)}\right] a_{0}^{\prime \prime} \\
& =(-1)^{q^{\prime \prime}-1} \gamma^{\mathcal{L}}\left(f_{0} e_{0}^{\prime \prime}\right)\left[\prod_{s=0}^{q^{\prime \prime}-1} \overline{\nu^{\prime \prime}(s)}\right] a_{0}^{\prime \prime} \\
& =\overline{\gamma\left(\vec{C}_{0}^{\prime \prime}\right)}\left(1-\overline{\gamma\left(\overrightarrow{C_{0}^{\prime \prime}}\right)}\right)^{-1} b_{0} .
\end{aligned}
$$

Hence, (3.18) holds for $p=1$ as well.

TheOREM 3.7. Let the core $\Theta_{e}=\left(B\left(q^{\prime}, 0, q^{\prime \prime}\right), \gamma_{\mid \vec{E}\left(B\left(q^{\prime}, 0, q^{\prime \prime}\right)\right)}\right)$ be a complex unit $\infty$-graph with two unbalanced cycles. Under the above notation (see also Fig. 3), for each non-zero complex number $a_{0}^{\prime}$, a generator $\mathbf{a}^{\prime}+\mathbf{a}^{\prime \prime}$ of the -2-eigenspace of $A\left(\mathcal{L}\left(\Theta_{e}\right)\right)$ is given by the formula

$$
a_{0}^{\prime \prime}=-\left(1-\overrightarrow{\gamma\left(\overrightarrow{C_{0}^{\prime \prime}}\right)}\right)^{-1}\left(1-\overrightarrow{\gamma\left(\overrightarrow{C_{0}^{\prime}}\right)}\right) \gamma^{\mathcal{L}}\left(e_{0}^{\prime \prime} e_{0}^{\prime}\right) a_{0}^{\prime},
$$

and

$$
\begin{array}{ll}
a_{i}^{\prime}=(-1)^{i}\left[\prod_{s=1}^{i} \overline{\nu^{\prime}(s)}\right] a_{0}^{\prime} & \text { for } 1 \leqslant i \leqslant q^{\prime}-1, \\
a_{i}^{\prime \prime}=(-1)^{i}\left[\prod_{s=1}^{i} \overline{\nu^{\prime \prime}(s)}\right] a_{0}^{\prime \prime} & \text { for } 1 \leqslant i \leqslant q^{\prime \prime}-1,
\end{array}
$$

where the $\nu^{\prime}(i)$ 's and the $\nu^{\prime \prime}(i)$ 's satisfy (3.11) and (3.13).

Moreover, $\mathbf{a}^{\prime}+\mathbf{a}^{\prime \prime}$ can be extended to a (-2)-eigenvector of $A(\mathcal{L}(\Phi))$ by putting zeros at all other entries.

Proof. Let $\nu^{\prime}(0)$ and $\nu^{\prime \prime}(0)$ be as in (3.14). In order to check that $A\left(\mathcal{L}\left(\Theta_{e}\right)\right)\left(\mathbf{a}^{\prime} \dot{+} \mathbf{a}^{\prime \prime}\right)=-2\left(\mathbf{a}^{\prime} \dot{+} \mathbf{a}^{\prime \prime}\right)$, it suffices to verify the eigenvalue equations at the vertices corresponding to the four edges incident to $v_{0}^{\prime}=v_{0}^{\prime \prime}$. Once again, by virtue of symmetry, we only consider the edge $e_{0}^{\prime}=v_{0}^{\prime} v_{1}^{\prime}$. We have to verify the equality

$$
-2 a_{0}^{\prime}=\nu^{\prime}(1) a_{1}^{\prime}+\overline{\nu^{\prime}(0)} a_{q^{\prime}-1}^{\prime}+\gamma^{\mathcal{L}}\left(e_{0}^{\prime} e_{0}^{\prime \prime}\right) a_{0}^{\prime \prime}+\gamma^{\mathcal{L}}\left(e_{0}^{\prime} e_{q^{\prime \prime}-1}^{\prime \prime}\right) a_{q^{\prime \prime}-1}^{\prime \prime} .
$$

This can be done once you observe that

$$
\begin{array}{cl}
\nu^{\prime}(1) a_{1}^{\prime}=-a_{0}^{\prime} & \text { by }(3.20) \text { when } i=1, \\
\overline{\nu^{\prime}(0)} a_{q^{\prime}-1}^{\prime}=-\overline{\gamma\left(\overrightarrow{C_{0}^{\prime}}\right)} a_{0}^{\prime} & \text { by }(3.15) \text { and }(3.20), \\
\gamma^{\mathcal{L}}\left(e_{0}^{\prime} e_{0}^{\prime \prime}\right) a_{0}^{\prime \prime}=-\left(1-\overrightarrow{\gamma\left(\overrightarrow{\left.C_{0}^{\prime \prime}\right)}\right.}\right)^{-1}\left(1-\overline{\gamma\left(\overrightarrow{C_{0}^{\prime}}\right)}\right) a_{0}^{\prime} & \text { by }(3.19),
\end{array}
$$


and

$$
\gamma^{\mathcal{L}}\left(e_{0}^{\prime} e_{q^{\prime \prime}-1}^{\prime \prime}\right) a_{q^{\prime \prime}-1}^{\prime \prime}=\left(1-\overrightarrow{\gamma\left(\overrightarrow{\left.C_{0}^{\prime \prime}\right)}\right.}\right)^{-1}\left(1-\overrightarrow{\gamma\left(\overrightarrow{C_{0}^{\prime}}\right)}\right) \overline{\gamma\left(\overrightarrow{C_{0}^{\prime \prime}}\right)} a_{0}^{\prime},
$$

which comes by (3.21), the equality $\gamma^{\mathcal{L}}\left(e_{0}^{\prime} e_{q^{\prime \prime}-1}^{\prime \prime}\right)=\gamma^{\mathcal{L}}\left(e_{0}^{\prime} e_{0}^{\prime \prime}\right) \nu^{\prime \prime}(0)$, and (3.15).

REMARK 3.8. If the two unbalanced cycles $C^{\prime}$ and $C^{\prime \prime}$ of a bicyclic core $\Theta_{e}$ have both gain -1 , Theorems 3.5, 3.6 and 3.7 return the same formulæ stated in Theorems 3.1, 3.3, and 3.5 in [5], where the -2-eigenvectors for signed line graphs are described.

For clarity, we recap the explained procedure for constructing an eigenbasis for -2 of $\mathcal{L}(\Phi)$ from the structure of the root graph $\Phi=(\Gamma, \gamma)$.

Step 1: Choose in $\Phi$ any connected line star complement, say $\Psi=\left(\Lambda, \gamma_{\mid \vec{E}(\Lambda)}\right)$.

Step 2: For each edge $e$ of $\Gamma$ not belonging to $\Phi$, form the one-edge extension $\Psi_{e}:=\left(\Lambda_{e}, \gamma_{\mid \vec{E}\left(\Lambda_{e}\right)}\right)$ of $\Psi$, where $V\left(\Lambda_{e}\right)=V(\Lambda)$ and $E\left(\Lambda_{e}\right)=E(\Lambda) \cup\{e\}$, and identify its core $\Theta_{e}$; the eigenvector $\mathbf{x}_{e}$ corresponding to $e$ is constructed by using an appropriate formula from one of Theorems 3.5, 3.6, and 3.7. These eigenvectors, if the $e$ 's are added in turn (one edge per each eigenvector), comprise an eigenbasis for -2 in $\mathcal{L}(\Phi)$.

We end this section by explaining how the -2-eigenspace of a complex unit line graph $\mathcal{L}(\Phi)$ changes when $\Phi$ is replaced by a switching equivalent graph. Let $\Phi_{1}=\left(\Gamma, \gamma_{1}\right)$ and $\Phi_{2}=\left(\Gamma, \gamma_{2}\right)$ be two complex unit gain graphs such that $\Phi_{2}=\Phi_{1}^{\zeta}$ for a suitable switching function $\zeta: V(\Gamma) \longrightarrow \mathbb{T}$, and let $H_{1}\left(\right.$ resp. $\left.H_{2}\right)$ be an incidence matrix of the complex unit gain graph $\Phi_{1}$ (resp. $\Phi_{2}$ ). By Proposition $2.13, D(\zeta)^{-1} \mathrm{H}_{1}$ is an incidence matrix for $\Phi_{2}$ such that $\mathcal{L}_{H_{1}}\left(\Phi_{1}\right)=\mathcal{L}_{D(\zeta)^{-1} \mathrm{H}_{1}}\left(\Phi_{2}\right)$. Hence, it follows from Proposition 2.10 that there exists a diagonal matrix $S$ such that $\mathrm{H}_{2}=D(\zeta)^{-1} \mathrm{H}_{1} S$. Finally, by Proposition 2.12 applied to $\Phi_{2}$, if $\mathbf{x}$ is an eigenvector of $A\left(\mathcal{L}_{H_{1}}\left(\Phi_{1}\right)\right)=A\left(\mathcal{L}_{D(\zeta)^{-1} \mathrm{H}_{1}}\left(\Phi_{2}\right)\right)$, then $S^{*} \mathbf{x}$ is an eigenvector of $A\left(\mathcal{L}_{H_{2}}\left(\Phi_{2}\right)\right)$.

4. Examples. In order to depict $\mathbb{T}$-gain graphs in Figs. 4 and 5, each continuous (resp., dashed) thick undirected line represents two opposite oriented edges with gain 1 (resp., -1), whereas the arrows detect the oriented edges $u v$ 's with an imaginary gain. The value $\gamma(u v)$ is specified near the correspondent arrow.
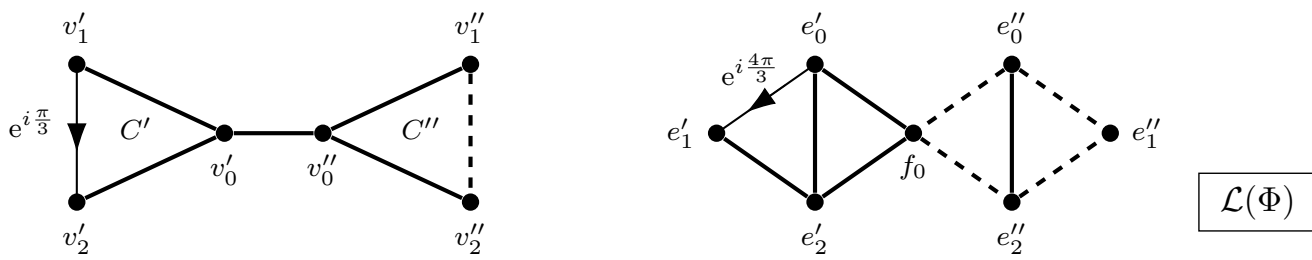

Figure 4. A complex unit dumbbell $\Phi$ and one of its associated line graphs $\mathcal{L}(\Phi)$.
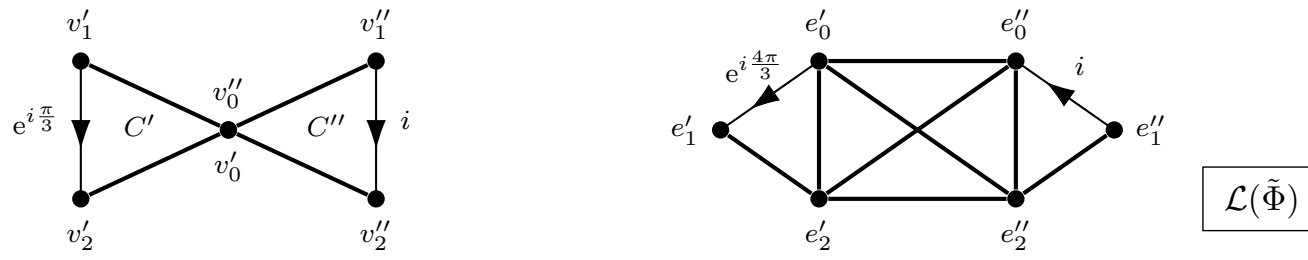

Figure 5. A complex unit $\infty$-graph $\tilde{\Phi}$ and one of its associated line graphs $\mathcal{L}(\tilde{\Phi})$. 
EXAMPLE 4.1. Let $\Phi=(\Gamma, \gamma)$ be the complex unit gain graph depicted in Fig. 4 . The vertex and the edge labeling are consistent with the one used in Fig. 2. Namely, $e_{i}^{\prime}=v_{i}^{\prime} v_{i+1}^{\prime}$ and $e_{i}^{\prime \prime}=v_{i}^{\prime} v_{i+1}^{\prime}$ for $i \in\{0,1\}$; $e_{2}^{\prime}=v_{2}^{\prime} v_{0}^{\prime}, e_{2}^{\prime \prime}=v_{2}^{\prime \prime} v_{0}^{\prime \prime}$, and $f_{0}=v_{0}^{\prime} v_{0}^{\prime \prime}$. In order to write down an incidence matrix $\mathrm{H}$ for $\Phi$ and the adjacency matrix of the corresponding line graph $\mathcal{L}(\Phi)$, we choose the ordering $v_{0}^{\prime}, v_{1}^{\prime}, v_{2}^{\prime}, v_{0}^{\prime \prime}, v_{1}^{\prime \prime}, v_{2}^{\prime \prime}$ for the elements in $V(\Gamma)$, and the ordering $e_{0}^{\prime}, e_{1}^{\prime}, e_{2}^{\prime}, f_{0}, e_{0}^{\prime \prime}, e_{1}^{\prime \prime}, e_{2}^{\prime \prime}$ for those in $E(\Gamma)$. The gains of the directed cycles $C_{0}^{\prime}:=e_{01}^{\prime} e_{12}^{\prime} e_{20}^{\prime}$ and $C_{0}^{\prime \prime}:=e_{01}^{\prime \prime} e_{12}^{\prime \prime} e_{20}^{\prime \prime}$ are

$$
\gamma\left(C_{0}^{\prime}\right)=\mathrm{e}^{i \frac{\pi}{3}} \quad \text { and } \quad \gamma\left(C_{0}^{\prime \prime}\right)=-1 .
$$

An incidence matrix $\mathrm{H}$ for $\Phi$ is given by

$$
\mathrm{H}=\left(\begin{array}{rrrrrrr}
1 & 0 & 1 & 1 & 0 & 0 & 0 \\
-1 & \mathrm{e}^{i \frac{\pi}{3}} & 0 & 0 & 0 & 0 & 0 \\
0 & -1 & -1 & 0 & 0 & 0 & 0 \\
0 & 0 & 0 & -1 & 1 & 0 & 1 \\
0 & 0 & 0 & 0 & -1 & 1 & 0 \\
0 & 0 & 0 & 0 & 0 & 1 & -1
\end{array}\right)
$$

According to the rules explained in Section 2.3, the graph $\mathcal{L}_{\mathrm{H}}(\Phi)$ is depicted in Fig. 4 and its adjacency matrix is

$$
A\left(\mathcal{L}_{\mathrm{H}}(\Phi)\right)=\left(\begin{array}{cccrrrr}
0 & \mathrm{e}^{i \frac{4 \pi}{3}} & 1 & 1 & 0 & 0 & 0 \\
\mathrm{e}^{i \frac{2 \pi}{3}} & 0 & 1 & 0 & 0 & 0 & 0 \\
1 & 1 & 0 & 1 & 0 & 0 & 0 \\
1 & 0 & 1 & 0 & -1 & 0 & -1 \\
0 & 0 & 0 & -1 & 0 & -1 & 1 \\
0 & 0 & 0 & 0 & -1 & 0 & -1 \\
0 & 0 & 0 & -1 & 1 & -1 & 0
\end{array}\right)
$$

For instance, $\gamma^{\mathcal{L}}\left(e_{0}^{\prime} e_{1}^{\prime}\right)=\bar{\eta}_{v_{1}^{\prime} e_{0}^{\prime}} \eta_{v_{1}^{\prime} e_{1}^{\prime}}=-\mathrm{e}^{i \frac{\pi}{3}}=\mathrm{e}^{i \frac{4 \pi}{3}}$. By Theorem 3.1 and Corollary 3.2, the graph $\Psi=$ $\Phi-\left\{e_{0}^{\prime}\right\}$ is a connected foundation, and $\Phi$ has the form $\Psi_{e_{0}^{\prime}}$. Hence, we expect to find -2 as eigenvalue of $A\left(\mathcal{L}_{H}(\Phi)\right)$ of multiplicity 1 . A MATLAB computation confirms that the characteristic polynomial

$$
\phi(\mathcal{L}(\Phi), x)=x^{7}-10 x^{5}-5 x^{4}+24 x^{3}+17 x^{2}-9 x-6,
$$

has seven distinct roots of multiplicity one, namely,

$$
\operatorname{Spec}\left(A\left(\mathcal{L}_{H}(\Phi)\right)\right)=\left\{-2,-\sqrt{3},-1,1-2 \cos \left(\frac{2 \pi}{9}\right), 1-2 \sin \left(\frac{\pi}{18}\right), \sqrt{3}, 1-2 \cos \left(\frac{2 \pi}{9}\right)\right\} .
$$

The row-column product confirms that the vector

$$
\left(a_{0}^{\prime}, a_{1}^{\prime}, a_{2}^{\prime}, b_{0}, a_{0}^{\prime \prime}, a_{1}^{\prime \prime}, a_{2}^{\prime \prime}\right)^{\top}=\left(2 \mathrm{e}^{i \frac{2 \pi}{3}}, 2 \mathrm{e}^{i \frac{\pi}{3}}, 2 \mathrm{e}^{i \frac{4 \pi}{3}}, 2,1,1,1\right)^{\top},
$$

is an -2-eigenvector for $A\left(\mathcal{L}_{H}(\Phi)\right)$. We leave to reader to check that its components satisfy the formulæ given in the statement of Theorem 3.6., after noting that

$$
\left(1-\overline{\gamma\left(\overrightarrow{C_{0}^{\prime}}\right)}\right)^{-1}=\mathrm{e}^{-i \frac{\pi}{3}}=-\mathrm{e}^{i \frac{2 \pi}{3}}
$$

EXAmple 4.2. Let $\tilde{\Phi}=(\tilde{\Gamma}, \tilde{\gamma})$ be the complex unit gain graph depicted in Fig. 5 . The vertex and the edge labeling are consistent with the ones used in Fig. 3. Namely, $v_{0}^{\prime}=v_{0}^{\prime \prime}, e_{i}^{\prime}=v_{i}^{\prime} v_{i+1}^{\prime}$, and $e_{i}^{\prime \prime}=v_{i}^{\prime \prime} v_{i+1}^{\prime \prime}$ for 
$i \in\{0,1\} ; e_{2}^{\prime}=v_{2}^{\prime} v_{0}^{\prime}$ and $e_{2}^{\prime \prime}=v_{2}^{\prime \prime} v_{0}^{\prime \prime}$. Once we choose the ordering $v_{0}^{\prime}, v_{1}^{\prime}, v_{2}^{\prime}, v_{1}^{\prime \prime}, v_{2}^{\prime \prime}$ for the elements in $V(\Gamma)$, and the ordering $e_{0}^{\prime}, e_{1}^{\prime}, e_{2}^{\prime}, e_{0}^{\prime \prime}, e_{1}^{\prime \prime}, e_{2}^{\prime \prime}$ for those in $E(\Gamma)$, an incidence matrix $\tilde{\mathrm{H}}$ for $\tilde{\Phi}$ is given by

$$
\tilde{\mathrm{H}}=\left(\begin{array}{rrrrrr}
1 & 0 & 1 & 1 & 0 & 1 \\
-1 & \mathrm{e}^{i \frac{\pi}{3}} & 0 & 0 & 0 & 0 \\
0 & -1 & -1 & 0 & 0 & 0 \\
0 & 0 & 0 & -1 & i & 0 \\
0 & 0 & 0 & 0 & -1 & -1
\end{array}\right)
$$

The graph $\mathcal{L}_{\tilde{\mathrm{H}}}(\tilde{\Phi})$ is depicted in Fig. 5 and its adjacency matrix is

$$
A\left(\mathcal{L}_{\tilde{\mathrm{H}}}(\tilde{\Phi})\right)=\left(\begin{array}{cccccc}
0 & \mathrm{e}^{i \frac{4 \pi}{3}} & 1 & 1 & 0 & 1 \\
\mathrm{e}^{i \frac{2 \pi}{3}} & 0 & 1 & 0 & 0 & 0 \\
1 & 1 & 0 & 1 & 0 & 1 \\
1 & 0 & 1 & 0 & -i & 1 \\
0 & 0 & 0 & i & 0 & 1 \\
1 & 0 & 1 & 1 & 1 & 0
\end{array}\right) .
$$

Note that the gains of the directed cycles $C_{0}^{\prime}:=e_{01}^{\prime} e_{12}^{\prime} e_{20}^{\prime}$ and $C_{0}^{\prime \prime}:=e_{01}^{\prime \prime} e_{12}^{\prime \prime} e_{20}^{\prime \prime}$ are

$$
\gamma\left(C_{0}^{\prime}\right)=\mathrm{e}^{i \frac{\pi}{3}} \quad \text { and } \quad \gamma\left(C_{0}^{\prime \prime}\right)=i
$$

In this example too, by Theorem 3.1 and Corollary 3.2, the graph $\tilde{\Psi}=\tilde{\Phi}-\left\{e_{0}^{\prime}\right\}$ is a connected foundation and $\tilde{\Phi}$ has the form $\tilde{\Psi}_{e_{0}^{\prime}}$. Hence, we expect to find -2 as eigenvalue of $A\left(\mathcal{L}_{\tilde{\mathrm{H}}}(\tilde{\Phi})\right)$ of multiplicity 1 . Our expectation is confirmed by a MATLAB computation, which gives

$$
\phi\left(\mathcal{L}_{\tilde{\mathrm{H}}}(\tilde{\Phi}), x\right)=(x+2)(x+1)(x-1)\left(x^{3}-2 x^{2}-5 x+3\right) .
$$

With hand calculations, it is not hard to verify that

$$
\mathbf{x}=\left(a_{0}^{\prime}, a_{1}^{\prime}, a_{2}^{\prime}, a_{0}^{\prime \prime}, a_{1}^{\prime \prime}, a_{2}^{\prime \prime}\right)^{\top}=\left(2,2 \mathrm{e}^{-i \frac{\pi}{3}}, 2 \mathrm{e}^{i \frac{2 \pi}{3}},(1-i) \mathrm{e}^{i \frac{4 \pi}{3}},(1+i) \mathrm{e}^{i \frac{\pi}{3}},(1+i) \mathrm{e}^{i \frac{4 \pi}{3}}\right)^{\top},
$$

is an -2-eigenvector for $A\left(\mathcal{L}_{\tilde{\mathrm{H}}}(\tilde{\Phi})\right)$. Its components satisfy the formulæ given in the statement of Theorem 3.7. In fact, since $\tilde{\gamma}^{\mathcal{L}}\left(e_{0}^{\prime \prime} e_{0}^{\prime}\right)=1$, for $a_{0}^{\prime}=2$, Equation 3.19 reads

$$
a_{0}^{\prime \prime}=-2\left(1-\overline{\gamma\left(\overrightarrow{C_{0}^{\prime \prime}}\right)}\right)^{-1}\left(1-\overrightarrow{\gamma\left(\overrightarrow{C_{0}^{\prime}}\right)}\right)=-2(1+i)^{-1}\left(1-\mathrm{e}^{-i \frac{\pi}{3}}\right)=(1-i) \mathrm{e}^{i \frac{4 \pi}{3}} .
$$

A simple check shows that the other components of $\mathbf{x}$ verify (3.20) and (3.21).

Acknowledgments. The authors thank the anonymous referee for his/her careful reading. His/her suggestions greatly improved the presentation of this paper. The research that led to the present paper was partially supported by a grant of the group GNSAGA of INdAM.

\section{REFERENCES}

[1] A. Alazemi, F. Belardo, M. Brunetti, M. Andelić, and C.M. da Fonseca. Line and subdivision graphs determined by $\mathbb{T}_{4}$-gain graphs. Mathematics, 7(10):926,2019.

[2] R.B. Bapat, D. Kalita, and S. Pati. On weighted directed graphs. Linear Algebra Appl., 436:99-111, 2012. 
[3] F. Belardo, M. Brunetti, M. Cavaleri, and A. Donno. Godsil-McKay switchings for mixed and gain graphs over the circle group. Linear Algebra Appl., (online 2020): DOI: 10.1016/j.laa.2020.04.025, in press.

[4] F. Belardo, M. Brunetti, and N. Reff. Balancedness and the least Laplacian eigenvalue of some complex unit gain graphs. Discuss. Math. Graph Theory, 40:417-433, 2020.

[5] F. Belardo, E.M. Li Marzi, and S.K. Simić. Signed line graphs with least eigenvalue -2: the star complement technique. Discrete Appl. Math., 207:29-38, 2016.

[6] F. Belardo, I. Sciriha, and S.K. Simić. On eigenspaces of some compound signed graphs. Linear Algebra Appl., 509:19-39, 2016.

[7] F. Belardo and S.K. Simić. On the Laplacian coefficients of signed graphs. Linear Algebra Appl., 475:94-113, 2015.

[8] F. Belardo, M. Brunetti, and A. Ciampella. Signed bicyclic graphs minimizing the least Laplacian eigenvalue. Linear Algebra Appl., 557:201-233, 2018.

[9] M. Cavaleri, D. D'Angeli, and A. Donno. A group representation approach to the balance of gain graphs, Journal of Algebraic Comb., in press, available at https://arxiv.org/abs/2001.08490.

[10] D. Cvetković, P. Rowlinson, and S. Simić. Eigenspaces of Graphs. Cambridge University Press, Cambridge, United Kingdom, 1997.

[11] D. Cvetković, P. Rowlinson, and S.K. Simić. Graphs with least eigenvalue -2 : The star complement technique. Journal of Algebraic Comb., 14:5-16, 2001.

[12] D. Cvetković, P. Rowlinson, and S. Simić. Spectral Generalizations of Line Graphs, On Graphs with Least Eigenvalue -2. Cambridge University Press, Cambridge, United Kingdom, 2004.

[13] D. Cvetković, P. Rowlinson, and S. Simić. Graphs with least eigenvalue -2: ten years on. Linear Algebra Appl., 484:504$539,2015$.

[14] K. Guo and B. Mohar. Hermitian adjacency matrix of digraphs and mixed graphs. J. Graph Theory, 85(1):217-248, 2017.

[15] R.A. Horn and C.R. Johnson. Matrix Analysis. Cambridge University Press, Cambridge, United Kingdom, 2012.

[16] D. Kalita. Properties of first eigenvectors and first eigenvalues of nonsingular weighted directed graphs. Electron. J. Linear Algebra, 30:227-242, 2015.

[17] N. Reff. Spectral properties of complex unit gain graphs. Linear Algebra Appl., 436(9):3165-3176, 2012.

[18] N. Reff. Oriented gain graphs, line graphs and eigenvalues. Linear Algebra Appl., 506:316-328, 2016.

[19] S. Simić, M. Anđelić, C.M. Da Fonseca, and D. Živković. On the multiplicities of eigenvalues of graphs and their vertex deleted subgraphs: old and new results. Electron. J. Linear Algebra, 30:85-105, 2015.

[20] Y. Wang, S.-C. Gong, and Y.-Z. Fan. On the determinant of the Laplacian matrix of a complex unit gain graph. Discrete Math., 341(1):81-86, 2018.

[21] T. Zaslavsky. Biased graphs. I: bias, balance, and gains. J. Combin. Theory Ser. B, 47:32-52, 1989.

[22] T. Zaslavsky. A mathematical bibliography of signed and gain graphs and allied areas. Electron. J. Combin., 5, 1998. Dynamic Surveys in Combinatorics, No. DS8 (electronic).

[23] T. Zaslavsky. Matrices in the Theory of Signed Simple Graphs. Advances in Discrete Mathematics and Applications, Mysore, 2008, in: Ramanujan Math. Soc. Lect. Notes Ser., vol. 13, Ramanujan Math. Soc., 207-229, 2010. 